\title{
Coupled electronic states in CdTe quantum dot assemblies fabricated by utilizing chemical bonding between ligands
}

Yong-Shin Lee, Tatsuya Ito, Kunio Shimura, Taichi Watanabe, Hang-Beom Bu, Kim Hyeon-Deuk, DaeGwi Kim

\begin{tabular}{|c|l|}
\hline Citation & Nanoscale. 12(13); 7124-7133 \\
\hline Issue Date & 2020-04-07 \\
\hline Type & Journal Article \\
\hline Textversion & Author \\
\hline Rights & $\begin{array}{l}\text { The following article has been accepted by Nanoscale. } \\
\text { This is the accepted manuscript version. Please cite only the published version. } \\
\text { The final, published version is available at https://doi.org/10.1039/D0NR00194E. }\end{array}$ \\
\hline Supplementary files & Supplementary information is available on https://doi.org/10.1039/D0NR00194E. \\
\hline DOI & 10.1039/D0NR00194E \\
\hline
\end{tabular}

Self-Archiving by Author(s)

Placed on: Osaka City University Repository

LEE Y.-S., ITO T., SHIMURA K., WATANABE T., BU H.-B., KIM D., \& HYEON-DEUK K. (2020). Coupled electronic states in CdTe quantum dot assemblies fabricated by utilizing chemical bonding between ligands. Nanoscale. 12, 7124-7133. DOI:10.1039/D0NR00194E 


\title{
Coupled electronic states in CdTe quantum dot
}

\section{assemblies fabricated by utilizing chemical bonding}

\section{between ligands}

Yong-Shin Lee ${ }^{1}$, Tatsuya Ito ${ }^{1}$, Kunio Shimura ${ }^{1}$, Taichi Watanabe ${ }^{1}$, Hang-Beom Bu ${ }^{1}$, Kim Hyeon-Deuk ${ }^{2}$, and DaeGwi Kim ${ }^{1 *}$

${ }^{1}$ Department of Applied Physics, Osaka City University, Osaka 558-8585, Japan

${ }^{2}$ Department of Chemistry, Kyoto University, Kyoto 606-8502, Japan

Keywords: Quantum dot, Superlattice, Quantum resonance, Miniband

\begin{abstract}
Semiconductor quantum dot superlattices (QDSLs) have attracted much attention as key materials for realizing new optoelectronic devices such as solar cells with high conversion efficiency and thermoelectric elements with high electrical conductivity. To improve the charge transport properties in the QDSL-based optoelectronic devices, it is important for the QD structures to form minibands, which are the coupled electronic states between QDs. A shorter inter-QD distance and a periodic arrangement of QDs are the essential conditions for the formation of minibands. In this
\end{abstract}


study, we use CdTe QDs capped with short ligands of N-acetyl-L cysteine (NAC) to fabricate a three-dimensional QD assemblies by utilizing the chemical bonding between NACs. The absorption spectra clearly display the quantum resonance phenomenon originating from the coupling of the wave functions between the adjacent QDs in the CdTe QD assemblies. Furthermore, we demonstrate the formation of minibands in the CdTe QD assemblies by examining both, the excitation energy dependence of the photoluminescence (PL) spectra and the detection energy dependence of the PL excitation spectra. The fabrication method of QD assemblies utilizing the chemical bonding between NACs can be applied to all QDs capped with NAC as a ligand.

\section{Introduction}

Semiconductor quantum dots (QDs) are known to exhibit unique quantum effects by confining carriers in nanometre-sized semiconductors. ${ }^{1}$ In QDs, relatively discrete energy states are formed similar to that of atoms, unlike continuous "energy bands" in bulk crystals, and the energy levels depend on the size of the QD. Owing to this, QDs are known as "artificial atoms" that offer control over the light absorption and the emission wavelength by changing the size of the QD. ${ }^{2}$ In addition, the surface passivation of QDs results in the high photoluminescence (PL) quantum yield, which can be used in various applications such as biomarkers ${ }^{3,4}$, displays ${ }^{5,6}$, and lasers. ${ }^{7}$ Discretization of energy states in QDs is considered to cause the phonon bottleneck ${ }^{8,9}$, which results in longer exciton relaxation times. This makes it possible to utilize QDs for hot electrons and multiple exciton generation (MEG), which are useful for the realization of solar cells with a high energy conversion efficiency. ${ }^{10}$ 
One of the most promising methods for synthesizing QDs is the colloidal method that utilizes a chemical reaction in a solution. The researchers have successfully synthesized QDs of various materials such as II-VI group of materials, $\mathrm{CdSe}^{11}, \mathrm{CdTe}^{12}$, and $\mathrm{ZnSe}^{13}$, I-III-VI group of materials, $\mathrm{CuInS}_{2}{ }^{14}$ and $\mathrm{AgInS}_{2}{ }^{15}$, and perovskite materials, $\mathrm{CH}_{3} \mathrm{NH}_{3} \mathrm{PbX}_{3}{ }^{16}$ and $\mathrm{CsPbX}_{3}{ }^{17}(\mathrm{X}=$ $\mathrm{Cl}, \mathrm{Br}$ and $\mathrm{I}$ ) using the colloidal method.

To date, the optical properties of colloidal QDs have been mainly studied by randomly dispersing QDs in a solution ${ }^{2,11,12,15}$. The dispersion of QDs in a solution allows a sufficiently large distance between the QDs such that there is no interaction between them. Therefore, most of the research on the optical properties of QDs is focused on the QDs as "isolated systems". In contrast, the system in which the QDs are closer and periodically arranged such as quantum dot superlattice (QDSL), the novel optical properties based on the interaction between the QDs should be observed. Specifically, "minibands", the coupled electronic states, can be formed in QDSLs ${ }^{18,19}$. The intermediate band solar cells using the minibands were proposed by Luque and Marti in $1997^{20}$ and Nozik in $2002 .{ }^{21}$ The theoretical studies predicted the energy conversion efficiency of the intermediate band solar cells to be approximately $60 \%$. In addition, the theoretical studies showed that the efficiency of MEG in the QDSLs is expected to be higher than the efficiency of the isolated QDs. ${ }^{22}$ Hence, the QDSL solar cells are expected to show a further improvement in its conversion efficiency. The formation of minibands in QDSLs also contributes to the improvement of the charge transport properties in electronic devices. In fact, QDSL-based field effect transistors ${ }^{23}$ and thermoelectric elements ${ }^{24}$ have been studied in recent years. So far, the miniband formation has been investigated mainly from the viewpoints of charge transport properties ${ }^{19,23,25,26}$ and theoretical calculations ${ }^{25,27}$. Little attention has been paid 
to direct observation of the miniband formation in terms of optical properties, especially PL properties.

The experimental procedure for fabricating QDSLs was first reported by Murray et al., who successfully fabricated SLs of colloidal CdSe QDs with excellent periodicity. ${ }^{28}$ Following this work, various methods for fabricating QDSLs such as drop casting ${ }^{29}$, spin $^{26}$ - or dip-coating ${ }^{30}$, and ligand exchange ${ }^{31}$ have been reported. Furthermore, some important advances in selfassembly of QDs and controlling the QD orientations and assembled structures have been reported very recently ${ }^{32-34}$.

A hot injection method utilizing a chemical reaction in organic solvents is generally used for the preparation of colloidal QDs ${ }^{35,36}$, and long-chain molecules such as trioctylphosphine oxide $(\mathrm{TOPO})^{35}$ and hexadecylamine (HDA) $)^{36}$ are typically used as ligands. Although the highly ordered QDSLs are fabricated using the QDs capped with long-chain ligands, the quantum resonance, which is the short-range coupling of wave functions between adjacent QDs $^{19,37,38}$ and the physical origin of the miniband formation in QDSLs, does not occur owing to the significantly long distance between the QDs. Furthermore, the wave function in CdSe/ZnS core/shell QDs is significantly attenuated by the potential barrier posed by the $\mathrm{ZnS}$ shell, which are used as model materials for conventional QD studies. ${ }^{11}$ Therefore, the minibands could not be observed in these QDSLs due to the absence of the quantum resonance.

In contrast, water-soluble QDs are often synthesized using short-chain ligands such as thioglycolic acid (TGA) ${ }^{12}$, mercaptopropionic acid (MPA) ${ }^{39}$, and N-acetyl-L cysteine (NAC) ${ }^{12,13}$. Since the lengths of these short-chain ligands are typically $0.4-0.6 \mathrm{~nm}$, which are much shorter than that of TOPO and HDA, the distance between the QDs can be significantly reduced in 
QDSLs using the water-soluble QDs, and the quantum resonance is expected to be induced. In fact, Kim et al. reported the quantum resonance phenomenon in the multilayers of the NACcapped CdTe QDs deposited by a layer-by-layer (LBL) assembly, originating from the coupling of wave functions between the adjacent QDs in lamination direction. ${ }^{38}$ In this paper, we report a fabrication method for three-dimensional (3D) CdTe QD assemblies that utilizes the chemical bonding between the ligands of NAC to achieve a remarkably short distance of approximately $0.9 \mathrm{~nm}$ between the adjacent QD surfaces. Furthermore, we present a direct spectroscopic evidence indicating the formation of minibands in the reported CdTe QD assemblies.

\section{Experimental}

\subsection{Synthesis of CdTe QDs}

NAC-capped CdTe QDs were prepared using a previously reported procedure. ${ }^{12}$ Firstly, $250 \mathrm{mg}$ of tellurium powder (purity 99.99\%; purchased from Kojundo Chemical) and $312.5 \mathrm{mg}$ of sodium borohydride (purity 98\%; purchased from Kanto Chemical) were put into a vial, and $6.0 \mathrm{~mL}$ of Deionized (DI) water was added and sealed. DI water was purged with $\mathrm{N}_{2}$ gas for 30 minutes before use. Since hydrogen gas is generated during the reaction, the lid of the vial was pierced with an injection needle for degassing. The vial was then cooled in an ice bath, and allowed to react for approximately 8 hours. In the solution, chemical reaction generates NaHTe as a tellurium ion source and $\mathrm{Na}_{2} \mathrm{~B}_{4} \mathrm{O}_{7}$ as a precipitate. Subsequently, NAC (purity 98\%; purchased from Kishida Chemical) and $\mathrm{Cd}(\mathrm{ClO} 4)_{2} \cdot 6 \mathrm{H}_{2} \mathrm{O}$ (purity 99\%; purchased from FUJIFILM Wako Pure Chemical) were dissolved in $100 \mathrm{~mL}$ of DI water. The solution was adjusted to $\mathrm{pH} 7.0$ by the stepwise addition of $0.2 \mathrm{mM} \mathrm{NaOH}$ (purchased from Kishida Chemical), and then the prepared NaHTe solution was 
added. Finally, dilute $\mathrm{HCl}$ (purchased from Kishida Chemical) was added and adjusted to $\mathrm{pH} 5.0$ to complete the CdTe QD precursor. The molar ratio in the precursor is $\mathrm{Cd}$ : Te: $\mathrm{NAC}=1.0: 0.3$ : 1.2 , and the concentration is $\left[\mathrm{Cd}^{2+}\right]=20 \mathrm{mM}$. CdTe QDs were synthesized by heating $10 \mathrm{~mL}$ of precursor in an autoclave at $200{ }^{\circ} \mathrm{C}$ for $10-40$ minutes and then cooled with an ice bath.

\subsection{Fabrication of CdTe QD assemblies}

The procedure for the fabrication of CdTe QD assemblies is discussed in the following paragraphs. Since the synthesized CdTe QD solution contains excess NAC-Cd complexes, they were removed using 2-propanol (purity 99.7\%; purchased from Kanto Chemical). CdTe QDs were precipitated by adding 2-propanol to the CdTe QD solution, and the solution was centrifuged at 3,500 rpm for 20 minutes. Thereafter, the supernatant containing NAC-Cd complexes was removed. Then, $\mathrm{CdTe}$ QDs were re-dispersed in $10 \mathrm{~mL}$ of DI water, and the QD solution was adjusted to $\mathrm{pH} 5.0$ by adding dilute $\mathrm{HCl}$. 1-ethyl-3-(3-dimethylaminopropyl) carbodiimide hydrochloride (EDAC) (purity 98\%; purchased from Kanto Chemical) was used for the amide bonds between NACs. 0.02 $\mathrm{g}$ of EDAC was dissolved in $10 \mathrm{~mL}$ of DI water and adjusted to $\mathrm{pH} 5.0$ using dilute $\mathrm{HCl}$. CdTe QD assemblies were fabricated by mixing the EDAC solution and the CdTe QD solution and stirring at $60^{\circ} \mathrm{C}$ for 120 minutes.

\subsection{Measurements}

Absorption spectra were measured using a JASCO V-650 spectrometer with a spectral resolution of $0.2 \mathrm{~nm}$. PL and PLE spectra were measured using a JASCO FP-8300 spectrofluorometer with a spectral resolution of $0.5 \mathrm{~nm}$. For the measurements of PL-decay profiles, a laser-diode ( $405 \mathrm{~nm}$, Hamamatsu PLP 10-040) with pulse duration of $100 \mathrm{ps}$ and a repetition of $100 \mathrm{kHz}$ was used as 
the excitation light source. The pump fluence was $50 \mathrm{~nJ} / \mathrm{cm}^{2}$. The PL-decay profiles were obtained by a time-correlated single-photon counting method. ${ }^{1} \mathrm{H}-\mathrm{NMR}$ spectroscopy was performed at 600 MHz on a Bruker Avance III HD 600. Fourier transform-infrared (FT-IR) spectra were measured using a JASCO FT/IR-430. Field emission scanning electron microscope (FE-SEM) image was obtained on a JEOL JSM-6500F. X-ray diffraction (XRD) measurements were performed with a Rigaku SmartLab using $\mathrm{Cu} \mathrm{K} \alpha$ radiation $(\lambda=0.154 \mathrm{~nm})$.

\section{Results \& discussion}

Figure 1a shows the structure of NAC that is used as a ligand for the current synthesis of the CdTe QDs. NAC is an organic molecule containing a $\mathrm{COOH}$ group and an $\mathrm{NH}$ group, and an amide bond between these two groups was used to fabricate QD assemblies. The bond length of NACs allows the distance between the surfaces of the adjacent QDs to be approximately $0.9 \mathrm{~nm}$, considering the bond lengths between the constituent elements such as S-C $(0.18 \mathrm{~nm}), \mathrm{C}-\mathrm{C}(0.15$ $\mathrm{nm}), \mathrm{C}-\mathrm{N}(0.14 \mathrm{~nm})$, and the bonding angle. Therefore, the QD assemblies prepared by this method are expected to form minibands owing to the possible short-range coupling of the wave functions between the adjacent QDs. To verify the role of the amide bonds between NACs as the driving force responsible for bringing QDs into proximity, we studied the energy transfer (ET) between the QDs.

Figure 1b shows the schematic ET process due to the proximity between the CdTe QDs of different diameters. We observed that ET occurs only when the QD distance is $10 \mathrm{~nm}$ or less ${ }^{40}$, and the ET rate is quite sensitive to the distance between the adjacent QDs. In addition, the experimental observation of PL can confirm the presence of the ET process by the PL quenching 
of the donor QDs (D-QDs) and increase in the PL signal of the acceptor QDs (A-QDs). The CdTe QDs with diameters $d=2.9 \mathrm{~nm}$ and $3.7 \mathrm{~nm}$ were used as the energy donors and energy acceptors, respectively. Figure 1c shows the absorption (dashed line) and the PL spectra (solid line) of the D-QDs (top) and A-QDs (bottom). Since the PL spectrum of the D-QDs and the absorption spectrum of the A-QDs sufficiently overlap, the ET between these QDs are expected to occur if these QDs are brought into close proximity by the amide bonds between NACs. Figure 1d shows the comparison of the PL spectra of the D- (circle) and A-QDs (rectangle) as well as the PL spectrum of the solution sample in which the two types of QDs are mixed (solid line). The identical PL spectral shape for each case confirms that the ET has not occurred. This result reflects that the distance between the QDs cannot be reduced simply by mixing the NACcapped QDs in a solution.

To promote the ET between the QDs, the sample was prepared by mixing the D-QD solution, the A-QD solution, and the condensing agent EDAC solution, and then stirring the mixture at $60{ }^{\circ} \mathrm{C}$ for $0-120 \mathrm{~min}$. Figure $2 \mathrm{a}$ and $\mathrm{b}$ show the dependence of the PL spectra and PL intensity on the duration of mixing (reaction time). The PL intensity of the D-QDs decreased, and conversely, the PL intensity of the A-QDs increased with an increase in the reaction time, indicating an ET from the D-QDs to the A-QDs. Figure 2c shows the time-dependent PL decay profiles detected at the PL peak energy of the D-QDs. The PL intensity decays faster with an increase in the reaction time. The PL decay profiles were fitted with three exponential functions: $I(t)=\sum A_{\mathrm{i}} \exp (-$ $\mathrm{t} / \tau_{\mathrm{i}}$ ), and the decay time $<\tau>$ was obtained from the average value of the three components: $\langle\tau>=$ $\sum A_{\mathrm{i}} \tau_{\mathrm{i}}{ }^{2} / \sum A_{\mathrm{i}} \tau_{\mathrm{i}}$, where $A_{\mathrm{i}}$ represents the respective weight of the components. Figure $2 \mathrm{~d}$ shows the dependence of $\langle\tau\rangle$ on the reaction time. The decrease in $\langle\tau\rangle$ with the reaction time is due to the ET from the D-QDs to the A-QDs. The experimental results in Figure 2 clearly demonstrate that 
an ET occurs from the D-QDs to the A-QDs. Additionally, these results confirm that utilizing the amide bonds between NACs is an effective approach for bringing QDs into close proximity.

In order to confirm the nature of the amide bonds between NACs, ${ }^{1} \mathrm{H}-\mathrm{NMR}$ and FT-IR measurements were performed on the samples before and after the reaction using the EDAC solution. A typical ${ }^{1} \mathrm{H}-\mathrm{NMR}$ spectrum of the NAC-capped CdTe QDs is shown in Figure 3a, which exhibits most of the resonance peaks in the range of $2.00-4.50 \mathrm{ppm}$. The upfield signal at 2.04 ppm corresponds to the methyl protons $\left(\mathrm{H}_{\mathrm{a}}\right)$. The methylene protons $\left(\mathrm{H}_{\mathrm{b}}\right)$ exhibit the NMR signals at $\sim 3.07-3.18 \mathrm{ppm}$, and the adjacent methine protons $\left(\mathrm{H}_{\mathrm{c}}\right)$ exhibit a signal at $4.49 \mathrm{ppm}$. The broadening of the proton signals at approximately $8.12 \mathrm{ppm}$ corresponds to the secondary amides $\left(\mathrm{H}_{\mathrm{d}}\right)$. This peak broadening is normally caused by the hydrogen bonding and the proton exchange. ${ }^{41}$ The relative areas of signals arising from the methyl $\left(\mathrm{H}_{\mathrm{a}}\right)$, methylene $\left(\mathrm{H}_{\mathrm{b}}\right)$, and methine $\left(\mathrm{H}_{\mathrm{c}}\right)$ protons are proportional to the number of hydrogen ions giving rise to each type of signal. For the amino proton $\left(\mathrm{H}_{\mathrm{d}}\right)$, the rapid exchange between deuterium in heavy water $\left(\mathrm{D}_{2} \mathrm{O}\right)$ and proton in the amide group can primarily contribute to a decrease in the integral area. ${ }^{42}$ Note that the expected carboxylic acid signal $\left(\mathrm{H}_{\mathrm{e}}\right)$ was not observed. The hydroxyl proton of the carboxylic acid displays a resonance signal significantly down-field (10.0-13.0 ppm) of other functions. A rapid exchange of these protons with deuterium in heavy water, as noted above, can cause the low-field signal to disappear. ${ }^{43}$

The inter-molecular cross-linking of NAC ligands by the amide bonding agent leads to a 3D network structure. The reaction is intensive and changes in the position of proton signals after cross-linking are seen in the obtained spectra. In the ${ }^{1} \mathrm{H}-\mathrm{NMR}$ spectra of cross-linking QDs, a complicated spectrum corresponding to the network structure was observed in Figure $3 \mathrm{~b}$ and $\mathrm{c}$. The methyl group exhibits peaks in the range of 1.85-2.25 ppm $\left(\mathrm{H}_{\mathrm{a}}, \mathrm{H}_{\mathrm{a}}\right.$ ' and $\left.\mathrm{H}_{\mathrm{a}}{ }^{\prime}\right)$, the methylene 
group shows the resonance peaks approximately in the range of 2.80-3.40 ppm $\left(\mathrm{H}_{\mathrm{b}}, \mathrm{H}_{\mathrm{b}}, \mathrm{H}_{\mathrm{b}}{ }^{\prime}\right)$, respectively. It is noteworthy that the signal intensity of the methine proton signals at approximately $4.45 \mathrm{ppm}\left(\mathrm{H}_{\mathrm{c}}, \mathrm{H}_{\mathcal{c}^{\prime}}\right.$ and $\left.\mathrm{H}_{\mathrm{c}^{\prime}}\right)$ showed a stepwise reduction. The electron withdrawing nature of the resultant carbonyl $(\mathrm{O}=\mathrm{C}-)$, amide $(\mathrm{O}=\mathrm{C}-\mathrm{N}-)$ and/or $\mathrm{N}, \mathrm{N}-$ dicarboxyamine group (-CO-N-CO-) can make alpha-hydrogen weakly acidic. ${ }^{44}$ Therefore, the alpha-hydrogen $\left(\mathrm{H}_{\mathrm{c}}, \mathrm{H}_{\mathrm{c}^{\prime}}\right.$ and $\left.\mathrm{H}_{\mathrm{c}}{ }^{\prime}\right)$ undergoes the hydrogen-deuterium exchange in $\mathrm{D}_{2} \mathrm{O}$, upon ingestion of $\mathrm{D}_{2} \mathrm{O}$. The spectra from the NAC cross-linking CdTe QD assemblies (Figure $3 \mathrm{~b}$ and c) evince a clear disappearance of the amino proton $\left(\mathrm{H}_{\mathrm{d}}\right)$ signals. The carbonyl group (NAC) was found to attach to another NAC ligand readily via the peptide bond formation with its secondary $\mathrm{N}-\mathrm{H}$ group, and the $\mathrm{C}-\mathrm{H}$ bond insertion to form $\mathrm{N}, \mathrm{N}-$ dicarboxyamine group. The hydrogen atoms on the nitrogen groups are abstracted from the NAC, and the spectrum resulted in a distinct signal loss of the nitrogen-proton $\left(\mathrm{H}_{\mathrm{d}}\right)$ intensity.

FT-IR spectra of the NAC-capped CdTe QDs and the NAC cross-linking CdTe QD assemblies are displayed in Figure 4. The major peaks observed in the NAC-capped CdTe QDs sample are discussed here. A wide band at the peak value of $3400 \mathrm{~cm}^{-1}$ corresponds to the stretching vibrations of the hydroxyl group [band of a free $\mathrm{OH}$ group (from water) and hydrogen bonds]. The negligible differences in the shape of this band observed before and after the cross-linking are owing to the participation of the -OH group in the hydrogen bonds. The N-H stretching vibrational modes $v(\mathrm{NH})$ of the amide group appears as a shoulder just below $3293 \mathrm{~cm}^{-1}$ in the broad envelope. ${ }^{45}$ Furthermore, the peak at $1633 \mathrm{~cm}^{-1}$ corresponds to the stretching vibrational modes of the carboxyamide group. ${ }^{46}$ The most significant bands at 1581 and $1392 \mathrm{~cm}^{-1}$ can be assigned to the asymmetric and symmetric stretching vibrational modes of the deprotonated carboxylic acid group $\left[v_{\text {as }}\left(\mathrm{COO}^{-}\right)\right.$and $\left.v_{\mathrm{s}}\left(\mathrm{COO}^{-}\right)\right]$, respectively. ${ }^{46}$ The band corresponding to the 
stretching vibrational modes of the $\mathrm{C}-\mathrm{O}$ bond and the deformation vibrations of the $\mathrm{C}-\mathrm{O}-\mathrm{H}$ bond $[v(\mathrm{C}-\mathrm{O})$ and $\delta(\mathrm{COH})]$ of the alcohol group occurs at the wave number of $1289 \mathrm{~cm}^{-1} \cdot{ }^{47}$

The major differences in the FT-IR spectra of the NAC cross-linking CdTe QD assemblies compared with that of the NAC-capped CdTe QDs are observed in the spectral range of 4000$500 \mathrm{~cm}^{-1}$ (Figure 4a and b). A dramatic decrease of the characteristic bands of $v(\mathrm{NH})$ of amide, $\left[v_{\text {as }}\left(\mathrm{COO}^{-}\right)\right.$and $\left.v_{\mathrm{s}}\left(\mathrm{COO}^{-}\right)\right]$of the deprotonated carboxylic acid, and $[v(\mathrm{C}-\mathrm{O})$ and $\delta(\mathrm{COH})]$ of the alcohol group, respectively, was observed. Additionally, some minor peak shifts have been observed in the spectrum of NAC cross-linking QD assemblies; for example, the stretching vibrational modes of the carboxyamide group at $1633 \mathrm{~cm}^{-1}$ shifts to $1636 \mathrm{~cm}^{-1}$. The results from this study are consistent with the ${ }^{1} \mathrm{H}-\mathrm{NMR}$ profile obtained before and after NAC cross-linking as discussed in the previous section.

Finally, we fabricated QD assemblies utilizing the amide bonds between QDs of equal diameters. Figure 5a shows the dependence of absorption (extinction) spectra on the reaction time. At zero minutes of reaction time (in CdTe QD solution), the clear absorption onset was observed at $\sim 1.95 \mathrm{eV}$, whereas the absorption tail was observed in the low-energy side of the spectrum for the reaction times of $30 \mathrm{~min}$ or longer. The absorption tail is mainly attributed to the light scattering by the QD assemblies, i.e., the spectra corresponding to the extinction spectra. Figure $5 \mathrm{~b}$ shows the dependence of the peak energy, estimated from the second derivative of the extinction spectra, on the reaction time. As the reaction time passes, the extinction peak gradually shifts to the low-energy side. The decrease in the peak energy occurs due to the quantum resonance between the adjacent CdTe QDs. Earlier, Kim et al. ${ }^{38}$ have reported the observation of the quantum resonance between the NAC-capped CdTe QD layers in 
the multilayers of QDs deposited by a LBL assembly. A cationic polyelectrolyte layer exists between the QD layers in such QD multilayer structures ${ }^{38}$ In this study, we directly use the chemical bonds formed between the ligands of NAC for bringing the QDs into proximity to fabricate the 3D "isotropic" QD assemblies with a short-range inter-QD distance. Therefore, the isotropic quantum resonance is expected owing to the close proximity between the QDs prepared by the method reported here. Figure $5 \mathrm{c}$ and $\mathrm{d}$ show the SEM image and the size histogram of the CdTe QD assemblies. The size analysis confirmed that the CdTe QD assemblies have an average size of $170 \mathrm{~nm}$.

Since minibands are the electronic states in which the lowest energy level of each QD is coupled by the quantum resonance, periodically ordered array structures of QDs are necessary. The XRD measurements were performed to confirm the periodicity of the present QD assemblies. Figure 6a shows the small-angle XRD patterns of the QD assemblies composed of CdTe QDs with the average diameters, $d=2.6,3.0,3.4$, and $3.7 \mathrm{~nm}$. For each QD assembly, a clear diffraction peak was observed at the small $2 \theta$ value $\left(\sim 2^{\circ}\right)$, indicating the nanoscale periodicity. The diffraction peaks observed at $2.5,2.3,2.1$, and $2.0^{\circ}$ indicate the structural regularity with the periodicities of $3.5,3.8,4.2$, and $4.4 \mathrm{~nm}$, respectively. These periods are in good agreement with the calculated values $(3.5,3.9,4.3$, and $4.6 \mathrm{~nm}$, respectively) obtained by the summation of $d$-values $(2.6,3.0,3.4$, and $3.7 \mathrm{~nm}$, respectively) and the cross-linking NAC length $(0.9 \mathrm{~nm})$. The agreement between the calculated and experimental values demonstrates that the periodic structural order is present in the formed assemblies of NAC-capped CdTe QDs.

Figure $6 \mathrm{~b}$ shows the dependence of the total energy shift $(\Delta E)$ of the extinction peak in the CdTe QD assemblies compared with the absorption peak of the CdTe QD solution (isolated QD system) as a function of the mean diameter of the CdTe QDs. As the QD diameter decreases, the 
value of $\Delta E$ increases. The increase in $\Delta E$ suggests an increase in the extent of the quantum resonance owing to the larger depth of the wave function penetration with the decrease in the QD size constituting the QD assemblies. ${ }^{38}$ Smaller the size of the QDs, stronger is the wave function coupling between the adjacent QDs, resulting in a stronger quantum resonance. ${ }^{38}$

In order to detect the formation of minibands as the coupled electronic states in the CdTe QD assemblies, the photoluminescence excitation (PLE) spectra were systematically investigated by changing the detection energy. Figure 7a shows the detection energy dependence of the PLE spectra of the CdTe QD solution (dispersion sample). The inset shows the PL spectrum of the same sample, and the down arrows in the figure indicate the corresponding detection energy in the PLE measurements. The PLE peak appears on the higher energy side with an increase in the detection energy. Such behaviour corresponds to that of the typical size-selective spectroscopy measurements ${ }^{48,49}$. The PLE peak observed on the high-energy side corresponds to the absorption by the small-sized QDs.

Figure 7b shows the schematic of the lowest-energy states in the QD-dispersed sample. The lowest-energy states in QDs are $\delta$-functional, and each QD has an intrinsic energy determined by the quantum size effect. In the conventional absorption and PL spectra, the spectra of QDs of different sizes have been observed as an ensemble; whereas, in the size-selective spectroscopy, a clear peak shift is observed because the QDs are selectively excited and detected based on their $\operatorname{sizes}^{48,49}$.

Figure 7c shows the detection energy dependence of the PLE spectra in the CdTe QD assemblies. Remarkably, the PLE peak energy does not change even though the detection energy is different, which is in contrast with the peak shifts in Figure 7a observed for the CdTe QD 
dispersion sample possessing the isolated electronic states. The disappearance of the size selectivity demonstrates that the observed PLE signal originates from the coupled electronic states. In periodical array structures of QDs, it is considered that the quantum resonance induced by the proximity of QDs forms an extended state (i.e. miniband) in which individual electronic states are coupled (Figure 7d). The quantum resonance has been discussed mainly from the point of view of the energy shift in the absorption spectra thus far. ${ }^{38}$ The PLE spectra observed in this study (Figure 7c) clearly show the experimental evidence of the formation of minibands in the QD assemblies.

Similar results were also observed in the size-selective PL measurements. Figure S1a and b (see supplementary information) show the excitation energy dependence of the PL spectra in the CdTe QD dispersion sample and the CdTe QD assemblies. The PL peak energy in the dispersion sample showed the shift in its position with the change in the excitation energy, whereas no dependence of the PL peak position on the excitation energy was again observed in the QD assemblies. We conclude that these experimental results are the clear evidence of the formation of coupled electronic states, i.e. minibands, in the CdTe QD assemblies.

\section{Conclusions}

In summary, we fabricated the 3D isotropic CdTe QD assemblies by utilizing the amide bonds between NACs that is used as a ligand. This method can be differentiated from the anisotropic layered structure fabricated by the previously reported LBL assembly. ${ }^{38}$ Our XRD measurements confirmed the periodic structural order of the CdTe QDs in the QD assemblies. The extinction peak in the CdTe QD assemblies was shifted to the lower-energy side compared with the 
absorption peak of the isolated CdTe QDs, which demonstrates the occurrence of the quantum resonance between the adjacent CdTe QDs. The dependence of the total energy shift on the QD diameter showed that the quantum resonance is more pronounced in the smaller QDs, reflecting the stronger resonant coupling. Moreover, the detection energy dependence of the PLE spectra and the excitation energy dependence of the PL spectra demonstrated the formation of coupled electronic states (minibands) in the CdTe QD assemblies. In recent years, various NAC-capped QDs such as $\mathrm{CdTe}^{12}, \mathrm{ZnSe}^{13}, \mathrm{ZnTe}^{50}$, and $\mathrm{CuInS}_{2}{ }^{51}$ with a high PL quantum yield have been successfully synthesized. The method proposed in this study will be suitable for fabricating QDSLs from such QDs employing NAC as a ligand. Furthermore, this method can be extended to the SLs prepared using metal, dielectric, and magnetic nanoparticles (NPs), and a hybrid structure formed by combining various types of NPs, and it is not limited only to semiconductor QDs. The present study opens avenues for exploring novel material design for various kinds of SLs using NPs which exhibit the strong quantum resonance.

\section{Author's contributions}

These authors contributed equally to this work.

\section{Conflicts of interest}

There are no conflicts to declare.

\section{Acknowledgements}

Y.-S.L. acknowledges financial support by JSPS KAKENHI Grant Number 19J13666. K.H.-D. acknowledges financial support from JST (PRESTO), Toyota Mobility Foundation, and Grantin-Aid for Scientific Research on Innovative Areas, Grant No. 18H05407. D.K. acknowledges 
financial support by JSPS Grant-in-Aids for Scientific Research from Japan Society for the Promotion of Science (KAKENHI), Grant Number 24560015.

\section{Supplementary Information}

Experimental data on the size-selective PL spectra are available from the Supplementary Information.

\section{References}

1. Y. Kayanuma, Phys. Rev. B, 1988, 38, 9797-9805.

2. C. B. Murray, D. J. Norris and M. G. Bawendi, J. Am. Chem. Soc., 1993, 115, 8706-8715.

3. X. Michalet, F. F. Pinaud, L. A. Bentolila, J. M. Tsay, S. Doose, J. J. Li, G. Sundaresan, A. M. Wu, S. S. Gambhir and S. Weiss, Science, 2005, 307, 538-544.

4. Q. Wang, Y. Xu, X. Zhao, Y. Chang, Y. Liu, L. Jiang, J. Sharma, D.-K. Seo and H. Yan, J. Am. Chem. Soc., 2007, 129, 6380-6381.

5. E. Jang, S. Jun, H. Jang, J. Lim, B. Kim and Y. Kim, Adv. Mater. (Winheim, Ger.), 2010, 22, 3076-3080.

6. T. Erdem and H. V. Demir, Nanophotonics, 2016, 5, 74-95.

7. V. I. Klimov, A. A. Mikhailovsky, S. Xu, A. Malko, J. A. Hollingsworth, C. A. Leatherdale, H.-J. Eisler and M. G. Bawendi, Science, 2000, 290, 314-317.

8. H. Benisty, C. M. Sotomayor-Torrès and C. Weisbuch, Phys. Rev. B, 1991, 44, 1094510948.

9. A. Pandey and P. Guyot-Sionnest, Science, 2008, 322, 929-932.

10. A. J. Nozik, M. C. Beard, J. M. Luther, M. Law, R. J. Ellingson and J. C. Johnson, Chem. 
Rev. (Washington, DC, U. S.), 2010, 110, 6873-6890.

11. D. V. Talapin, A. L. Rogach, A. Kornowski, M. Haase and H. Weller, Nano Lett., 2001, 1, $207-211$.

12. H.-B. Bu, H. Kikunaga, K. Shimura, K. Takahashi, T. Taniguchi and D. Kim, Phys. Chem. Chem. Phys., 2013, 15, 2903-2911.

13. Y.-S. Lee, K. Nakano, H.-B. Bu and D. Kim, Appl. Phys. Express, 2017, 10, 065001.

14. L. Li, A. Pandey, D. J. Werder, B. P. Khanal, J. M. Pietryga and V. I. Klimov, J. Am. Chem. Soc., 2011, 133, 1176-1179.

15. T. Uematsu, K. Wajima, D. K. Sharma, S. Hirata, T. Yamamoto, T. Kameyama, M. Vacha, T. Torimoto and S. Kuwabata, NPG Asia Mater., 2018, 10, 713-726.

16. F. Zhang, H. Zhong, C. Chen, X.-G. Wu, X. Hu, H. Huang, J. Han, B. Zou and Y. Dong, ACS Nano, 2015, 9, 4533-4542.

17. L. Protesescu, S. Yakunin, M. I. Bodnarchuk, F. Krieg, R. Caputo, C. H. Hendon, R. X. Yang, A. Walsh and M. V. Kovalenko, Nano Lett., 2015, 15, 3692-3696.

18. O. L. Lazarenkova and A. A. Balandin, J. Appl. Phys. (Melville, NY, U. S.), 2001, 89, 55095515.

19. C. R. Kagan and C. B. Murray, Nat. Nanotechnol., 2015, 10, 1013-1026.

20. A. Luque and A. Marti, Phys. Rev. Lett., 1997, 78, 5014-5017.

21. A. J. Nozik, Phys. E (Amsterdam, Neth.), 2002, 14, 115-120.

22. I-Y. Chang, D. Kim and Kim H.-D., J. Phys. Chem. C, 2019, 123, 2549-2556.

23. J.-H. Choi, A. T. Fafarman, S. J. Oh, D.-K. Ko, D. K. Kim, B. T. Diroll, S. Muramoto, J. G. Gillen, C. B. Murray and C. R. Kagan, Nano Lett., 2012, 12, 2631-2638.

24. D. Yang, C. Lu, H. Yin and I. P. Herman, Nanoscale, 2013, 5, 7290-7296. 
25. K. Whitham, J. Yang, B. H. Savitzky, L. F. Kourkoutis, F. Wise and T. Hanrath, Nat. Mater., $2016,15,557-563$.

26. J.-S. Lee, M. V. Kovalenko, J. Huang, D. S. Chung and D. V. Talapin, Nat. Nanotechnol., $2011,6,348-352$.

27. J. C. Ondry, J. P. Philbin, M. Lostica, E. Rabani and A. P. Alivisatos, ACS Nano, 2019, 13, $12322-12344$.

28. C. B. Murray, C. R. Kagan and M. G. Bawendi, Science, 1995, 270, 1335-1338.

29. D. V. Talapin and C. B. Murray, Science, 2005, 310, 86-89.

30. E. A. Gaulding, B. T. Diroll, E. D. Goodwin, Z. J. Vrtis, C. R. Kagan and C. B. Murray, $A d v$. Mater. (Winheim, Ger.), 2015, 27, 2846-2851.

31. P. R. Brown, D. Kim, R. R. Lunt, N. Zhao, M. G. Bawendi, J. C. Grossman and V. Bulović, ACS Nano, 2014, 8, 5863-5872.

32. Y. Nagaoka, R. Tan, R. Li, H. Zhu, D. Eggert, Y. A. Wu, Y. Liu, Z. Wang and O. Chen, Nature, 2018, 561, 378-382.

33. Y. Nagaoka, H. Zhu, D. Eggert and O. Chen, Science, 2018, 362, 1396-1400.

34. H. Zhu, Z. Fan, L. Yu, M. A. Wilson, Y. Nagaoka, D. Eggert, C. Cao, Y. Liu, Z. Wei, X. Wang, J. He, J. Zhao, R. Li, Z. Wang, M. Grünwald and O. Chen, J. Am. Chem. Soc., 2019, 141, 6013-6021.

35. X. Peng, J. Wlickham and A. P. Alivisatos, J. Am. Chem. Soc., 1998, 120, 5343-5344.

36. S. R. Cordero, P. J. Carson, R. A. Estabrook, G. F. Strouse and S. K. Buratto, J. Phys. Chem. $B, 2000, \mathbf{1 0 4}, 12137-12142$.

37. T. Hanrath, J. Vac. Sci. Technol., A, 2012, 30, 030802.

38. D. Kim, S. Tomita, K. Ohshiro, T. Watanabe, T. Sakai, I-Y. Chang and Kim H.-D. Nano 
Lett., 2015, 15, 4343-4347.

39. S. F. Wuister, I. Swart, F. Driel, S. G. Hickey and C. M. Donegá, Nano Lett., 2003, 3, 503507.

40. D. Kim, S. Okahara, M. Nakayama and Y. Shim, Phys. Rev. B, 2008, 78, 153301.

41. M. P. Williamson, Prog. Nucl. Magn. Reson. Spectrosc., 2013, 73, 1-16.

42. C. Boyer and T. P. Davis, Chem. Commun. (Cambridge, U. K.), 2009, 6029-6031.

43. D. E. Metzler, C. M. Metzler, E. T. Mollova, R. D. Scott, S. Tanase, K. Kogo, T. Higaki and Y. Morino, J. Biol. Chem., 1994, 269, 28017-28026.

44. J. M. Chalker, S. B. Gunnoo, O. Boutureira, S. C. Gerstberger, M. Fernández-González, G. J. L. Bernardes, L. Griffin, H. Hailu, C. J. Schofield and B. G. Davis, Chem. Sci., 2011, 2, $1666-1676$.

45. B. Koleva, M. Spiteller and T. Kolev, Amino Acids, 2010, 38, 295-304.

46. I. Dolamic, C. Gautier, J. Boudon, N. Shalkevich and T. Bürgi, J. Phys. Chem. C, 2008, 112, $5816-5824$.

47. J. F. Pearson and M. A. Slifkin, Spectrochim. Acta, part A, 1972, 28, 2403-2417.

48. P. A. M. Rodrigues, G. Tamulaitis, P. Y. Yu and S. H. Risbud, Solid State Commun., 1995, 94, 583-587.

49. J. Miyazaki and S. Kinoshita, Phys. Rev. B, 2012, 86, 035303.

50. H.-B. Bu and D. Kim, Chem. Lett., 2018, 47, 152-155.

51. K. Iida, Y. Uehigashi, H. Ichida, H.-B. Bu and D. Kim, Bull. Chem. Soc. Jpn., 2019, 92, 930-936. 


\section{Figures}

a

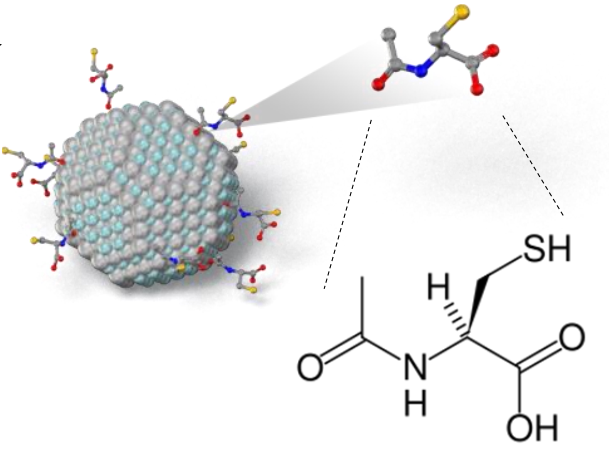

c

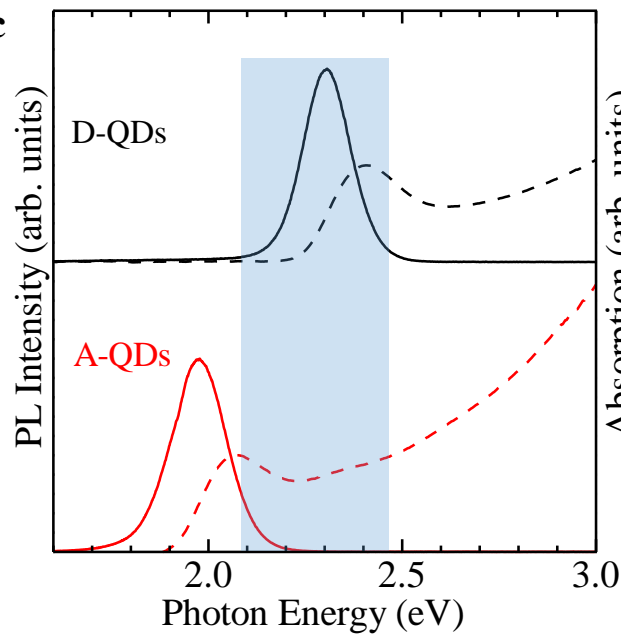

b

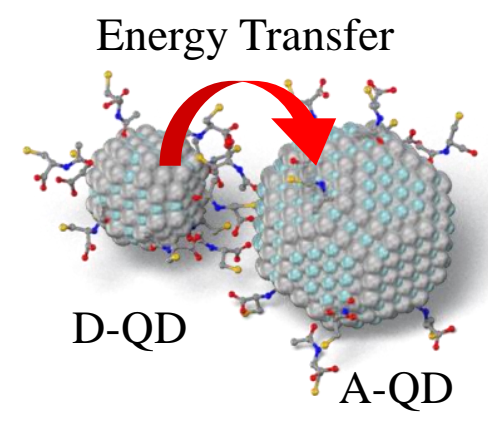

d

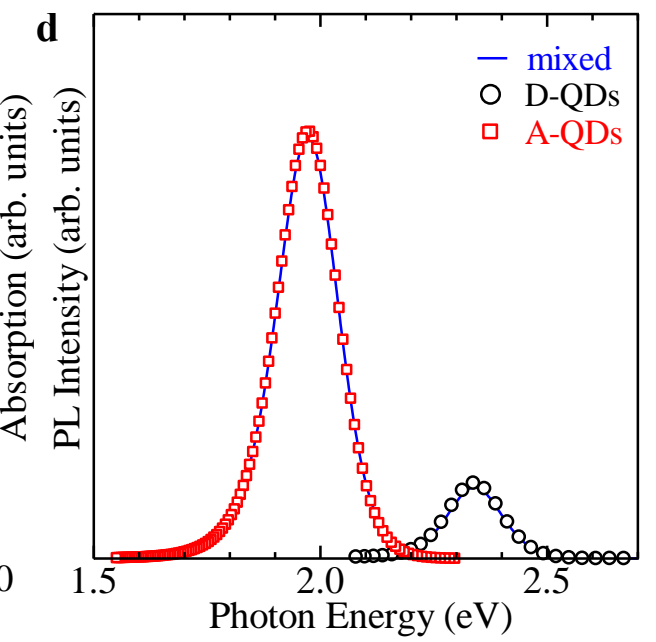

Fig. 1 (a) Structure of NAC used as a ligand. (b) Energy transfer process in the NAC cross-linking QDs of different sizes. D-QD (A-QD) represents an energy donor (acceptor) with a small (large) QD. (c) Absorption (dashed line) and PL spectra (solid line) of D-QDs (top) and A-QDs (bottom). The shaded rectangle represents the spectral overlap region between the PL of the D-QDs and the absorption of the A-QDs. (d) PL spectra of D-QDs (black circle), A-QDs (red rectangle), and the solution in which the two types of QDs are simply mixed (solid line). 

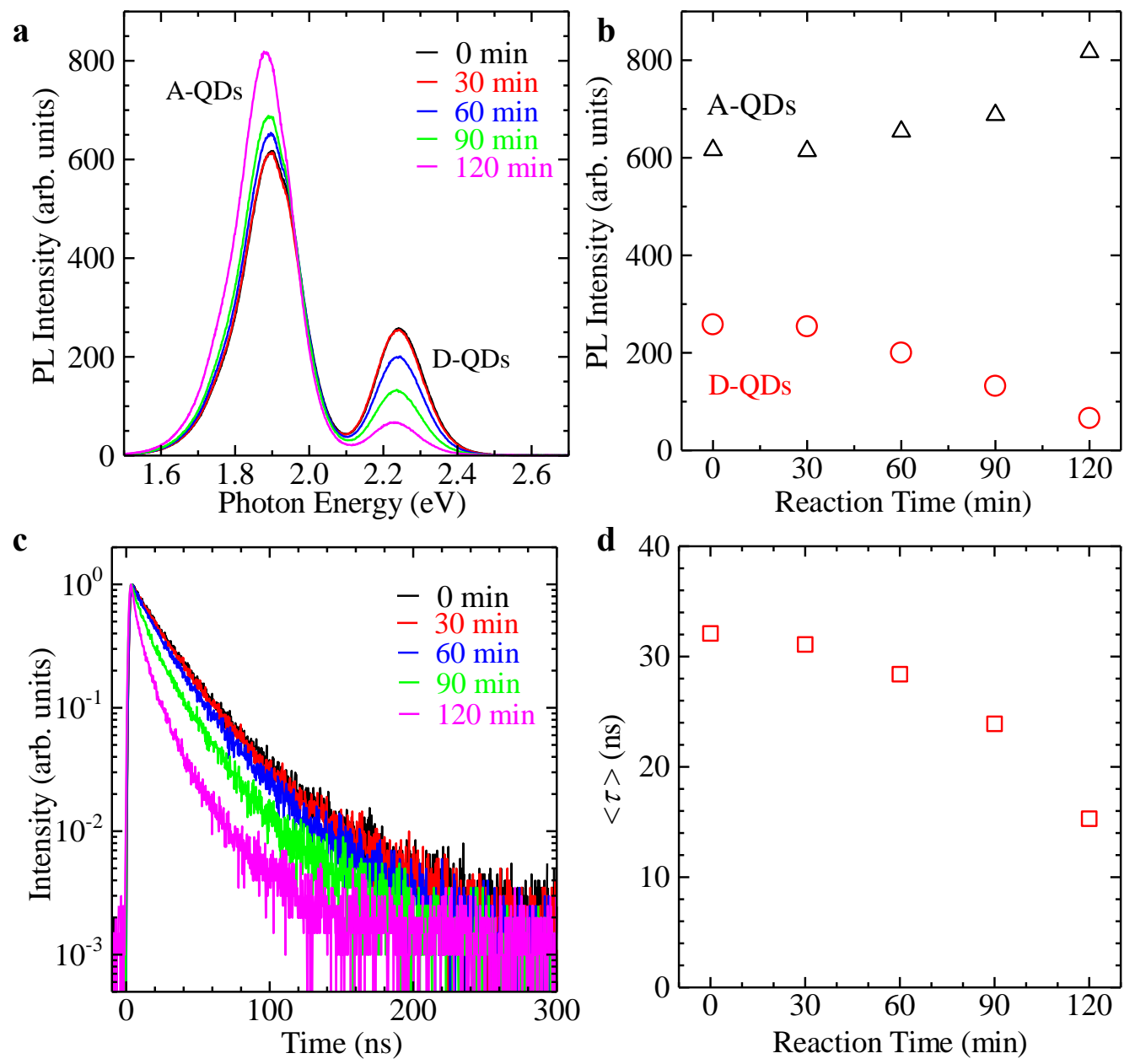

Fig. 2 Demonstration of QD proximity effect by amide bonds based on Fluorescence Resonance Energy Transfer (FRET) observations. (a) Dependence of the PL spectra on the reaction time. (b) Dependence of the PL intensity on the reaction time. (c) PL decay profiles detected at the PL peak energy of D-QDs. (d) Dependence of the average decay time $\langle\tau\rangle$ on the reaction time. 
$\mathbf{a}$

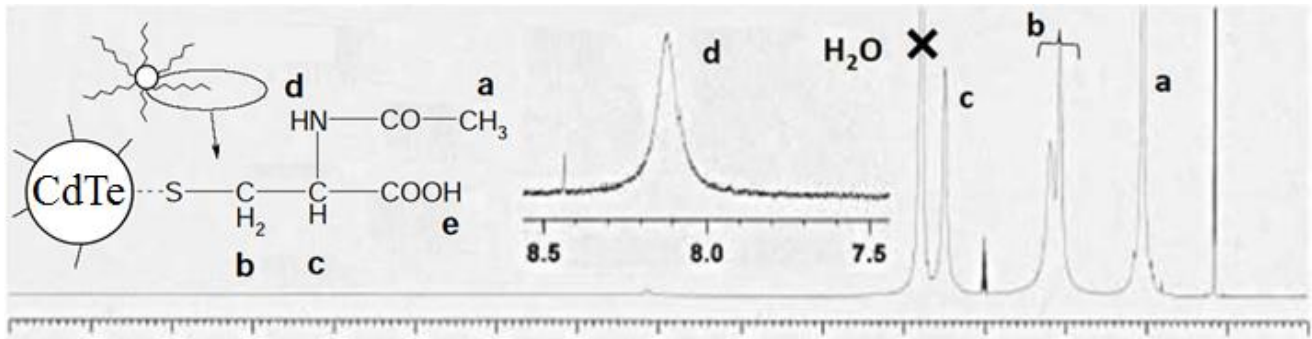

b
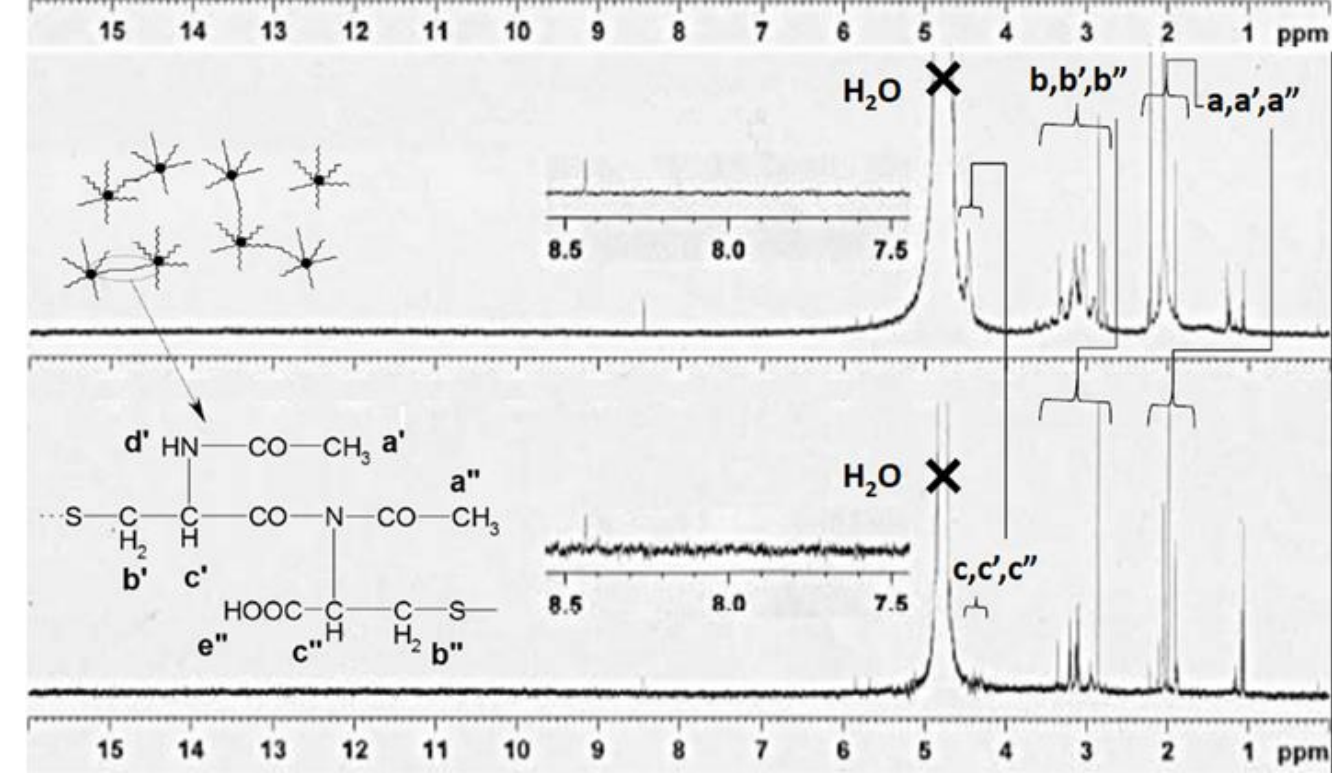

Fig. 3 Demonstration of amide bonds between NACs by ${ }^{1} \mathrm{H}-\mathrm{NMR}$ analysis. (a) NAC-capped CdTe QDs. (b,c) NAC cross-linking CdTe QD assemblies. 

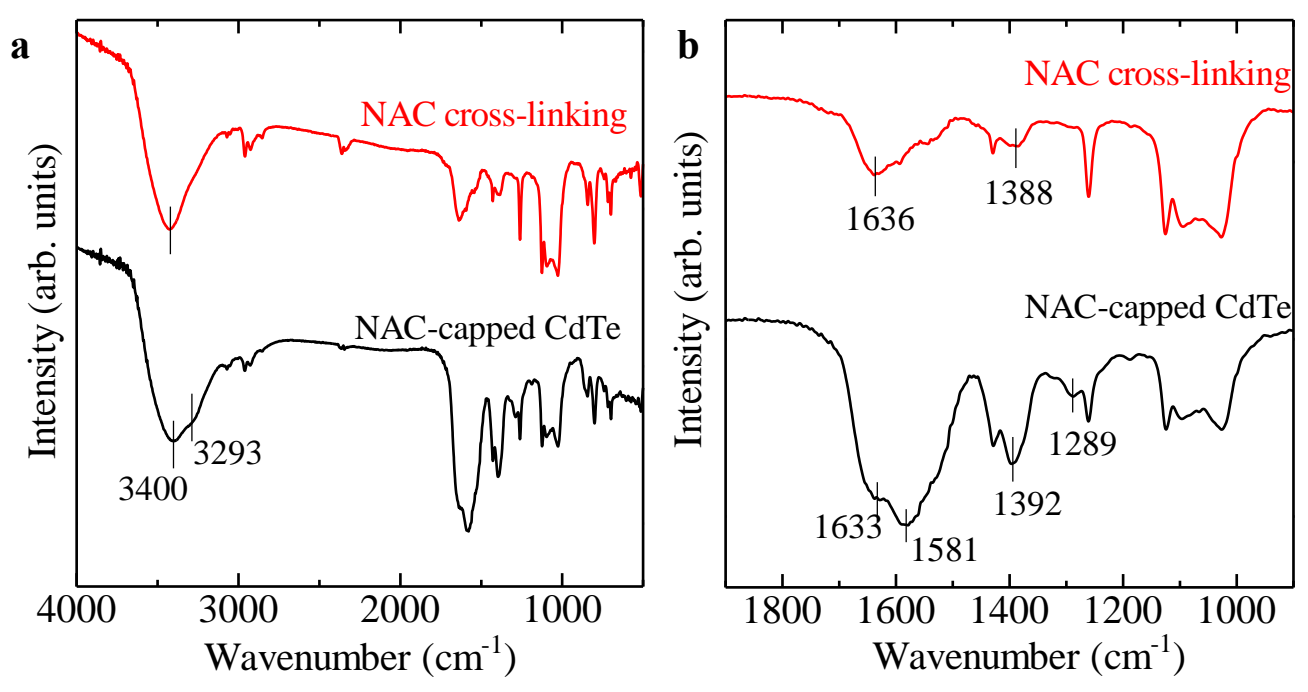

Fig. 4 Demonstration of amide bonds between NACs by FT-IR analysis. (a) FT-IR spectra of the NAC-capped CdTe QDs (bottom) and the NAC cross-linking CdTe QD assemblies (top) of fullscale. (b) Magnified spectra in the range of $1900-900 \mathrm{~cm}^{-1}$. 

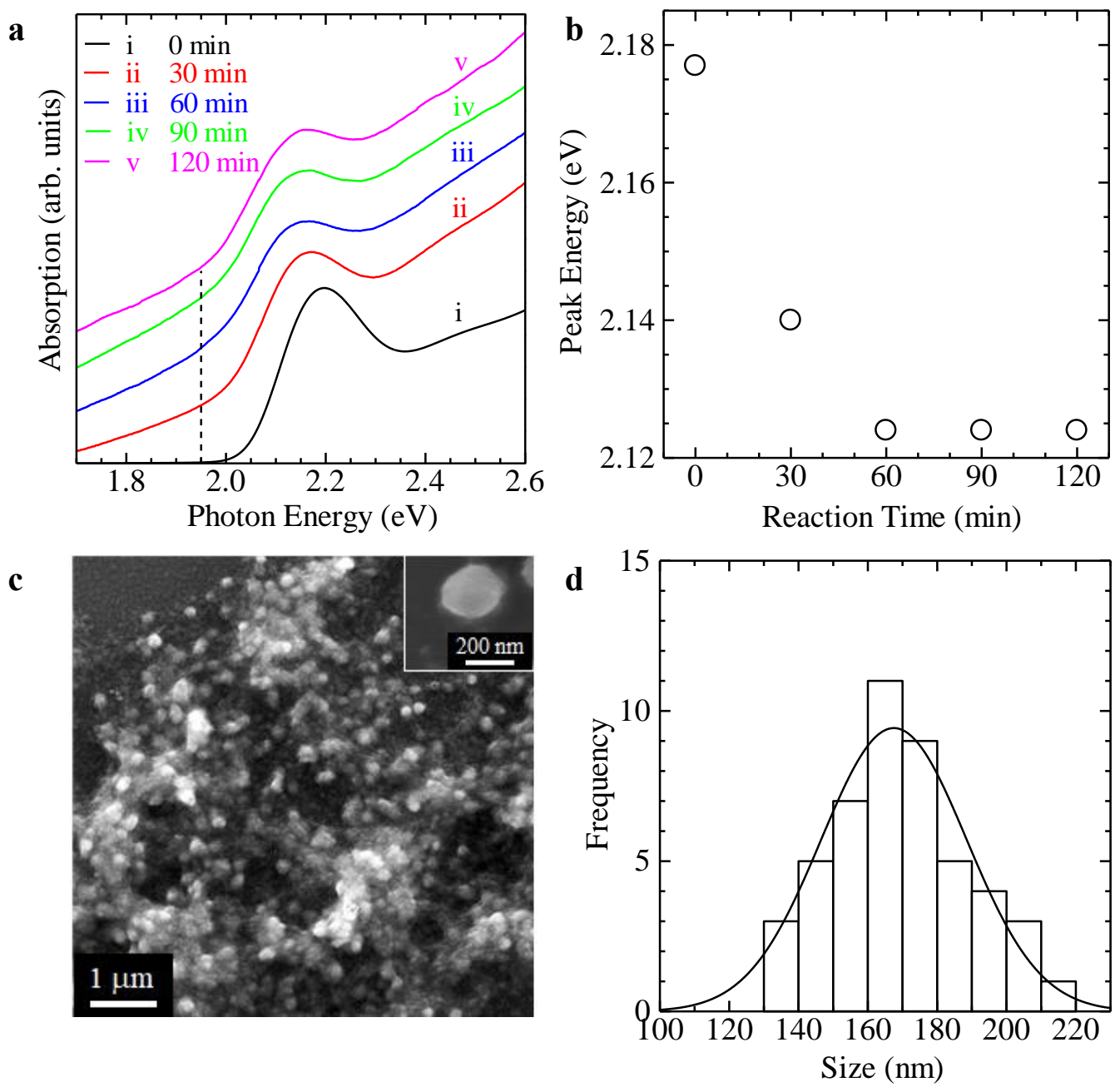

Fig. 5 Observation of quantum resonance in CdTe QD assemblies. (a) Dependence of the absorption (extinction) spectra on the reaction time. Here, 0 min represents the absorption spectrum of the CdTe QD solution and 30-120 min represent the extinction spectra of the CdTe QD assemblies. (b) Dependence of the extinction peak energy on the reaction time. (c) SEM image of the CdTe QD assemblies. The inset shows magnified SEM image. (d) Size histogram of the CdTe QD assemblies. The solid curve represents a gaussian fitting function with an average size of $173 \mathrm{~nm}$ and a standard deviation of $22 \mathrm{~nm}$. 

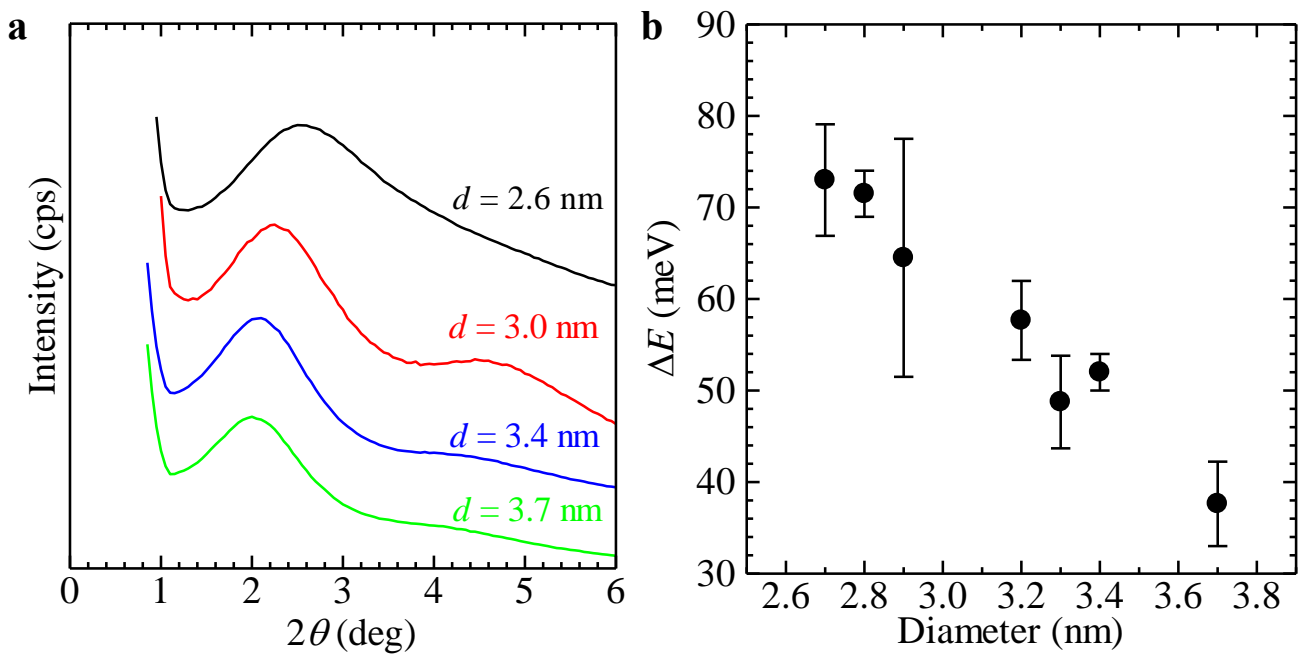

Fig. 6 Demonstration of periodicity in QD assemblies. (a) Small-angle XRD patterns for CdTe QD assemblies fabricated by using different-sized CdTe QDs with the diameters of $d=2.6,3.0$, 3.4, and $3.7 \mathrm{~nm}$. (b) Dependence of the total energy shift $(\Delta E)$ on the diameter of the CdTe QDs. Closed circles and error bars represent the average value and standard deviation of the total energy shift. 
a

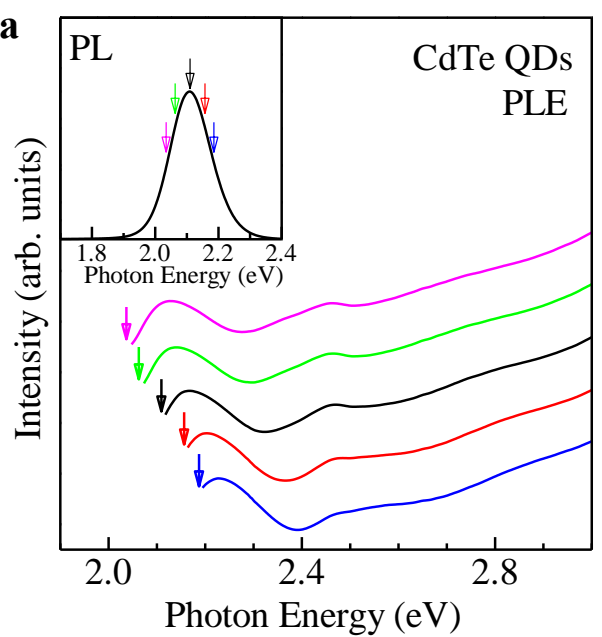

c

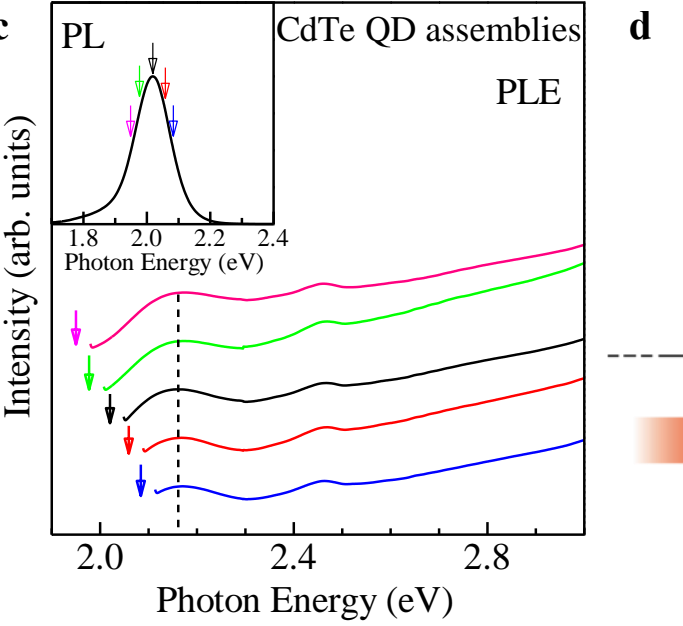

b
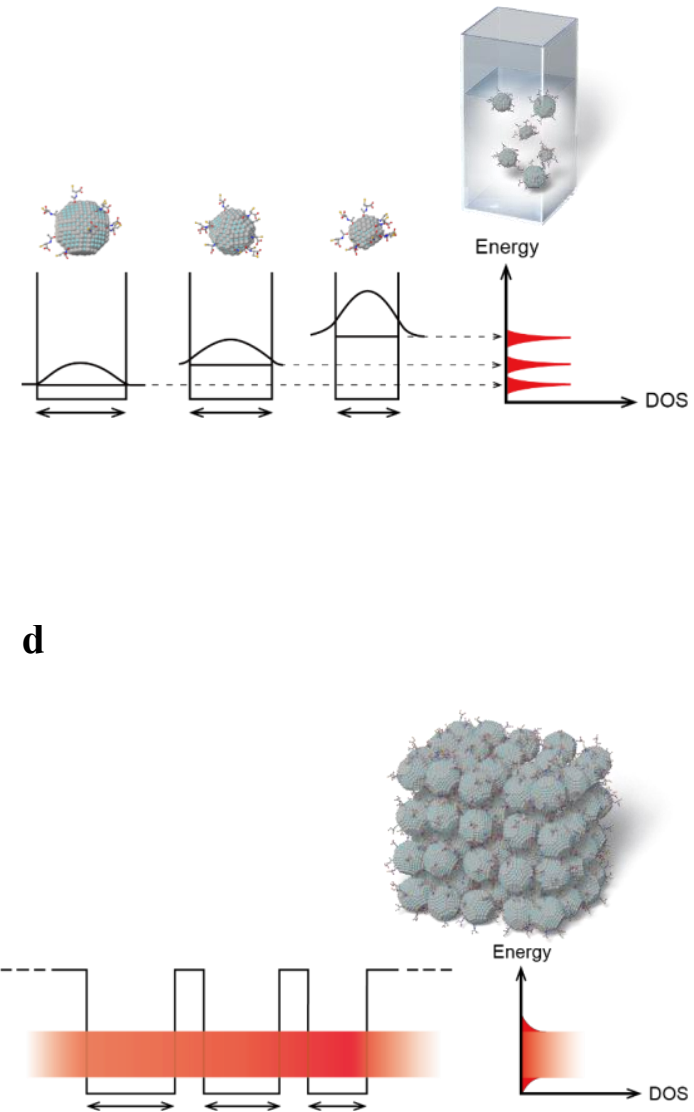

Fig. 7 Size selective spectroscopy. (a) Detection energy dependence of the PLE spectra obtained for the CdTe QD solution (dispersion sample). The inset shows the PL spectrum of the same sample. The down arrows indicate the detection energy in the PLE measurements. (b) Schematic of the lowest-energy levels in the CdTe QD solution. (c) Detection energy dependence of the PLE spectra obtained for the CdTe QD assemblies. The dashed vertical line represents the PLE peak energy (2.16 eV). (d) Schematic of the lowest-energy levels for the CdTe QD assemblies. 